\title{
Antecedentes de las sociedades cooperativas en México
}

\author{
Carlos Muñiz Díaz y \\ J. Dolores Alanís Tavira² \\ Universidad Autónoma del Estado de México
}

Sumario: Introducción. I. El sistema cooperativo en México. II. De sociedades mutualistas a sociedades cooperativas. III. El cooperativismo durante el porfiriato. IV. Ley General de Sociedades Cooperativas del 15 de febrero de 1938. V. Cooperativismo en México después del cardenismo. VI. Conclusiones. VII. Bibliografía.

Resumen. El presente trabajo tiene como objetivo analizar históricamente el desarrollo de las cooperativas en México, tomando en cuenta los principales momentos que ha vivido nuestro país, la época prehispánica, la colonia, la independencia, la revolución y la época postrevolucionaria. La metodología aplicada principalmente es a través del método histórico, que es un procedimiento de estudio de los fenómenos culturales que nos permite estudiar los hechos del pasado con el fin de encontrar explicaciones causales a las manifestaciones propias de las sociedades actuales, buscando reconstruir el pasado de la manera más objetiva posible.

Palabras clave: Antecedentes, Cooperativas, México.

Abstract. The present work aims to historically analyze the development of cooperatives in Mexico, taking into account the main moments that our country has lived, the pre-Hispanic era, the colony, independence, the revolution and the post-revolutionary era. The methodology applied mainly is through the historical method, which is a study procedure of cultural phenomena that allows us to study the events of the past in order to find causal explanations for the manifestations of current societies, seeking to reconstruct the past of the as objective as possible.

Keywords: Background, Cooperatives, Mexico.

1 Doctor en Derecho. Investigador del Centro de Investigación en Ciencias Jurídicas, Justicia Penal y Seguridad Pública. UAEMex. Miembro del Sistema Nacional de Investigadores Nivel I.

2 Doctor en Derecho. Director de la Facultad de Derecho de la Universidad Autónoma del Estado de México. 


\section{Introducción}

El presente trabajo, tiene como objetivo analizar históricamente el desarrollo de las cooperativas en México, tomando en cuenta los principales momentos que ha vivido nuestro país, la época prehispánica, la colonia, la independencia, la revolución y la época postrevolucionaria. La metodología aplicada principalmente es a través del método histórico que es un procedimiento de estudio de los fenómenos culturales que nos permite estudiar los hechos del pasado con el fin de encontrar explicaciones causales a las manifestaciones propias de las sociedades actuales, buscando reconstruir el pasado de la manera más objetiva posible. ${ }^{3}$

Las sociedades cooperativas tienen su origen en la búsqueda histórica que ha tenido la humanidad para obtener una sobrevivencia con el fin y objetivo de resolver los problemas que presenta la vida en sociedad.

Todas las sociedades han creado instrumentos de cooperación y de asociación para darle vida y permanencia armónica a la comunidad y una armonía entre los medios de producción y el capital contra casi siempre la fuerza de trabajo, fuerzas contrarias que le han dado forma y sentido a la legislación laboral en general.

El termino cooperatio nos comenta Luis Inostroza Fernández 4 «ignifica realizar una actividad en común. Esta actividad se daba en las comunidades primitivas en la forma de cooperación simple, en la que participaban las personas mayores en el marco de una división de trabajo que definía las tareas de recolección de alimentos, defensa y organización interna. Además, se sustentaba en la propiedad colectiva de los medios de producción».

Después se da la época feudal en la que este sistema de organización social necesita fortalecerse contra los señores feudales y los emperadores que como sistema abusaban de las clases desprotegidas esclavizándolas, las clases nobles se dedicaban a mantener el poder político y económico a costa de los siervos y esclavos y así se defendía el Estado Absolutista.

Más adelante el Socialismo Utópico ${ }^{5}$ se constituyó en una filosofía con otra línea de pensamiento donde propugnaba el ideal de una sociedad libre e igualitaria con la meta de la justicia social involucrando

3 Dzul, Escamilla, Mariela, "Aplicación Básica de los métodos científicos», Universidad Autónoma del Estado de Hidalgo, http://www.uaeh.edu.mx/virtual.

4 Inostroza Fernández, Luis, Movimiento cooperativista internacional. Cooperativismo y sector social en México, México, Universidad Autónoma Metropolitana, 1989.

5 El Socialismo Utópico escuela de pensamiento francés, crítico de la propiedad privada. 
a los sectores campesinos tan desfavorecidos durante la época feudal. Los Filósofos Utopistas (siglo XVI y XVII) hicieron una crítica principalmente a ese abuso de las clases trabajadoras y campesinas y soñaron por una sociedad más justa, los principales filósofos fueron:

- Thomas Moro (1478-1535).

- Tommaso Campanella (1568-1639).

- Francis Bacon (1561-1626).

- James Harrington (1611-1677).

Estos autores planteaban la idea de una sociedad basada en la asociación libre de los productores, no tenía argumento que los que trabajan dependieran de los que no lo hacen, es decir se basaba en principios de economía socialista de producción y del consumo colectivo.

Los filósofos cooperativistas se dirigieron a influenciar una serie de ideologías y escuelas de pensamiento que afectó los movimientos políticos los cuales le atribuyen a estas ideas gran importancia en sus fundamentos y en sus estatutos.

El estado de Bienestar derivado de la teoría del «Estado Protector» le debe al cooperativismo su estructura científica y filosófica en la que su base argumentativa es buscar el bien común, que se conjunte con un sistema de gobierno que no es el comunismo del siglo XX, sino producto de una democracia que respete las libertades y las individualidades del hombre.

\section{El sistema cooperativo en México}

Es importante comentar que el imperio Azteca tuvo o generó una forma de producción cooperativa en varios aspectos, los principales fueron: la educación, defensa, salud, construcción tanto de sus templos como de sus palacios. Quizá los Calpulli, se consideró el núcleo estructural de la sociedad azteca, porque a partir de esta forma de organización, los aztecas se defendían, se educaban, sembraban sus tierras y se relacionaban en comunidad. ${ }^{6}$ Contaban con una forma de gobierno autosuficiente que respetaba a su "Consejo de Ancianos», generando una estructura social definida que daba como resultado unidad social, étnica, religiosa, espiritual, etc.

6 Izquierdo, Martha E. Comentarios en torno a las sociedades cooperativas en México, Boletín de la Asociación Internacional de Derecho Cooperativo, Bilbao, España. Universidad de Deusto, Núm. 46/2012. 
También El Calpulli Azteca tenía una organización política que se dedicaba al control del pago de tributos de los pueblos que iban conquistando eso daba concepto del poder que podían ejercer a favor de la propia comunidad azteca.

El Calpulli organizaba el trabajo y la vida en común que se llevaba a cabo en las comunidades buscando la solidaridad y la cooperación, con la característica que la tenencia de la tierra era comunal y estaba prohibida su venta, nadie podía considerase como dueño o propietario individual. Una circunstancia en materia laboral prehispánica es que se consideraba que si alguien faltaba al trabajo sin causa justificada, perdía el usufructo de la parcela un antecedente que aún recogen las leyes laborales contemporáneas como causal te recisión de la relación laboral, se entiende como una primitiva semejanza.

Posteriormente con la llegada de los españoles a México en 1521, toda esta organización comunal y cooperativa tuvo un gran cambio en la organización y el sistema productivo, la colonización se realizó de forma militar, Luis Inistroza ${ }^{7}$ nos explica cuáles fueron las medidas compulsivas:

- Despojo y apropiación de tierras, valles, minas y otros recursos productivos de los indígenas.

- Organización y creación de pueblos especialmente los indios (conformación de las reducciones indígenas, que posteriormente dieron lugar a las comunidades).

- Imposición de formas de administración y de gobierno de corte hispano-europeo en las comunidades indígenas.

- Presión e imposición de ideas religiosas como base para imponer su ideología.

Estas medidas compulsivas alteraron las formas comunales y cooperativas de la vida de los pueblos prehispánicos, así los municipios y ciudades fueron consumiendo a los callpullis aunque se les respeto algunos territorios que se pudieron mantener en propiedad comunal.

\section{De sociedades mutualistas a sociedades cooperativas}

El referente más antiguo de las cooperativas en México, lo encontramos en el periodo colonial con los gremios y las cofradías. Los primeros consistían en agrupaciones de artesanos jerárquicamente organi-

7 Inostroza Fernández, Luis, op. cit. p. 65 
zados; aunque estos no surgieron para dar respuesta a las necesidades de los grupos de artesanos, más bien fueron traídas desde España, a la Nueva España para organizar el trabajo y la producción. Las segundas eran asociaciones de socorro mutuo que se constituían en torno a actividades religiosas, pero tenían fondos de beneficencia que prestaban auxilio a sus integrantes en caso de enfermedad, accidente, muerte entre otros aspectos. ${ }^{8}$

La revolución industrial iniciada en Inglaterra a finales del siglo XVIII, que se extendería durante la primera parte del siglo XIX a gran parte de Europa occidental, trajo consigo transformaciones económicas, tecnológicas y sociales; se pasó de una economía rural basada fundamentalmente en la agricultura y el comercio a una economía de carácter urbano, industrializada y mecanizada. Por un lado, se dio la concentración de capital en unas cuantas personas y mano de obra libre que se vio obligada a vender su fuerza de trabajo. Por otro lado, la introducción de maquinaria motriz para la producción en serie para el mercado, que orilló a la competencia entre los productores. En este proceso, aquellos productores sin capacidad de competencia, como los artesanos y campesinos, se vieron desplazados y forzados a incorporarse al grupo de trabajadores asalariados. ${ }^{9}$

En México desde el fin de la independencia (1821) y hasta 1860 se experimentó un moderado crecimiento en los sectores minero y agrícola. Respecto al primer sector, entre 1821 y 1850 llegaron a México compañías inglesas, alemanas y estadounidenses para hacer grandes inversiones de capital en las explotaciones mineras; en cuanto el segundo sector, las haciendas y ranchos experimentaron un crecimiento, sobretodo, en la producción de azúcar. ${ }^{10}$

El sector que más creció en México fue el industrial en especial el ramo textil, la producción de tejidos de lana y algodón fue la principal rama industrial. La mayor parte de la producción textil se realizaba en los lugares tradicionales, el obraje, el taller artesanal, así como talleres domésticos. Entre 1830 y 1845 se dio el desarrollo de la una industria algodonera moderna con la llegada de maquinaria barata de Estados

8 Olvera López, Adriana, «El Sistema Cooperativo Industrial Mexicano: una revisión histórica 1929-1958», Tesis de Licenciatura en Economía, México, UNAM, 2001, p. 28.

9 Galindo Merchant, Agripina, Las sociedades cooperativas en México: algunas observaciones sobre su funcionamiento, México, Universidad Autónoma Metropolitana Unidad Azcapotzalco, 1987, p. 1.

10 Sánchez Santiró, Ernest, «El desempeño de la economía mexicana, 1810-1860: de la colonia al Estado-Nación», en Sandra Kuntz Ficker (coordinadora), Historia económica general de México. De la colonia a nuestros días, México, El Colegio de México, Secretaría de Economía, 2010, pp. 285, 288. 
Unidos y la contratación de técnicos especializados extranjeros. No obstante, este desarrollo perdería dinamismo en los años cincuenta, y solo a partir de 1880 volvería a experimentar un acelerado crecimiento. ${ }^{11}$ Durante este periodo el proletariado industrial como tal no existía aún en México, más bien era una clase que apenas iniciaba su formación con el establecimiento de las primeras industrias.

Los campesinos, ante la imposibilidad de competir con los grandes empresarios, empezaron a asociarse para contrarrestar los efectos negativos que el desarrollo industrial provocó en su economía. El inicio del movimiento de los trabajadores lo llevaron a cabo los oficiales, maestros de los gremios y propietarios de pequeños talleres que fueron paulatinamente desplazados por la industria, que podría considerarse «moderna». Por esta razón las primeras asociaciones de trabajadores son de carácter gremial y mutualista. ${ }^{12}$

Al mismo tiempo que se daban el desarrollo de la industria en México (1830-1845), en Europa surgían distintas corrientes teóricas que proponían formas de organización para contrarrestar los efectos propiciados por el capitalismo. Se considera que el cooperativismo es un sistema económico que nace en Inglaterra durante la década de 1820 a partir de que los trabajadores buscan superar las condiciones sociales en las que se llevan a cabo sus actividades de producción. Varios autores consideran que la Cooperativa de Rochdale, formada en 1844 por 28 obreros, fue el primer ejercicio de cooperativismo en el mundo. ${ }^{13}$

Entre las distintas propuestas teóricas surgidas en Europa en contra del capitalismo estaban las de los comunistas, anarquistas y utópicos. Los primeros proponían una transformación radical de los medios de producción, los segundos consideraban que el problema se resolvería con la eliminación del Estado y los terceros proponían crear colonias comunitarias para distribuir el producto en función del trabajo aportado por cada uno de los integrantes. ${ }^{14}$

El fracaso de estas teorías obligo a buscar formas de organización más factibles y reales, de esta forma empezaron a surgir las sociedades

11 Sánchez Santiró, Ernest, «El desempeño de la economía mexicana, 1810-1860: de la colonia al Estado-Nación», en Sandra Kuntz Ficker (coordinadora), op. cit. pp. 289-290

12 Olvera López, Adriana, op. cit., p. 30.

13 Centro de Estudios Sociales y de Opinión Pública, "Cooperativismo en México», Cámara de Diputados, 2016, p. 5. Recuperado de file:///C:/Users/HP/Documents/Derecho/Servicio\%20social/Bibliografia_Coperativas/CESOP-IL-14-DT217CooperativismosEnMexico-160628.pdf (fecha de consulta: 01 de octubre de 2020)

14 Galindo Merchant, Agripina, op. cit., p. 2. 
de crédito, ahorro y consumo que tenían como objetivo principal cubrir las necesidades materiales inmediatas de sus agremiados. Este tipo de sociedades surgieron a mediados del siglo XIX en Francia, Alemania e Inglaterra. ${ }^{15}$ Estas ideas sobre sociedades mutualistas empezaron a ser adoptadas en México, por lo que campesinos y artesanos de distintos ramos empezaron a asociarse para mejorar su situación frente al avance del capitalismo; incluso, los gobiernos mexicanos vieron en este tipo de asociaciones una manera de que el país se desarrollara económicamente.

El 10 de septiembre de 1846 Manuel Crescencio Rejón, ministro de relaciones exteriores, reconoce el derecho de los ciudadanos para asociarse y el 1 de febrero de 1856 Ignacio Comonfort publica el decreto sobre establecimiento de colonias agrarias. El reconocimiento del derecho de asociación fue un gran avance, ya que permitió a los grupos movilizarse y lograr, por ejemplo, en Guadalajara la fundación de una Sociedad de Artesanos en 1850 y el 5 de junio de 1853 en la Ciudad de México crear la Sociedad Particular de Socorros Mutuos, siendo sus miembros un grupo de sombrereros. ${ }^{16}$

De esta forma, enmarcado dentro del periodo de Reforma, surge el mutualismo que en un inicio solo plantea la defensa del proletariado y del artesano urbano de la competencia. El derecho de asociación adquirió rango constitucional al establecerse en el artículo 9 de la Constitución Política de 1857 que:

«A nadie se le puede coartar el derecho de asociarse o de reunirse pacíficamente con cualquier objeto licito; pero solamente los ciudadanos de la Republica puede hacerlo para tomar parte en los asuntos políticos del país. Ninguna reunión armada tiene derecho a deliberar». ${ }^{17}$

No obstante, las inversiones realizadas en México durante la primera mitad del siglo XIX en la minería e industria, la economía estaba sustentada en las estructuras agrarias; por lo mismo el sistema de producción estaba basado en la agricultura. En este sentido, las ideologías europeas sobre las formas de organización tuvieron mayor éxito y difusión entre los campesinos que entre la clase obrera; la cual estaba en formación.

El interés por el asociacionismo se incrementó con la llegada a México del griego Plutino Rhodeakanati en 1861, quien tomó la pro-

15 Ibidem, p. 4.

16 Olvera López, Adriana, op. cit., p. 31.

17 Gobierno de México, Constitución Federal de los Estados Unidos Mexicanos de 1857, México, Imprenta de F. Guzmán y Hermanos, 1875. 
puesta del gobierno mexicano establecida en el decreto del 1 de febrero de 1856 donde se hacía una invitación a los extranjeros para residir en el país otorgarles la nacionalidad. Rhodeakanati era de ideas socialistas y un ferviente promotor de las sociedades agrícolas utópicas; de hecho, un año antes de que llegara a México ya se había formado la primera sociedad agrícola.

Si bien, el asociacionismo tuvo mayor éxito entre los campesinos; los trabajadores también empezaron a conformarse en sociedades mutualistas, por ejemplo, en 1864 algunos sombrereros de la Ciudad de México formaron una sociedad mutualista. Al mismo tiempo en Europa se consolidaban las sociedades cooperativas como una forma de organización distinta a las mutualidades, las existencias de estas formas de organización empezaron a difundirse en América Latina y a influenciar a los líderes anarquistas, quienes buscaban darle un giro a las sociedades agrícolas y mutualistas, para convertirlas en sociedades cooperativas. ${ }^{18}$

En México se tenían informes vagos e imprecisos del cooperativismo europeo; fue hasta 1868 que se conoce plenamente el funcionamiento interno y los éxitos económicos del cooperativismo impulsados en Francia, Alemania e Inglaterra. De esta forma, en la década de 1870 empezaron a surgir una serie de organizaciones encaminadas a la ayuda mutua y la defensa de sus intereses comunes, como las cajas de ahorro, sociedades agrícolas y sociedades de socorros mutuos. Estas sociedades, aunque no tenían un conocimiento claro de lo que era el cooperativo se caracterizaban por la solidaridad, pudiéndolas ubicar así en una etapa de precooperativismo. ${ }^{19}$ Así surgieron varias sociedades, por ejemplo, en 1871 se conforma la Sociedad Cooperativa «Unión y Progreso» perteneciente al ramo de rebosaría. El 3 de marzo de 1872 se trasformó la Mutua Sociedad Progresista de Carpinteros en sociedad cooperativa recibiendo el nombre oficial de «Compañía Cooperativa de Obreros de México» y a finales de dicho mes se presentaron las bases constitutivas de la sociedad; no obstante, al igual que el taller cooperativo, las divisiones entre los dirigentes provocaron su cierre. ${ }^{20}$

18 Galindo Merchant, Agripina, op. cit., p. 10.

19 Méndez Montoya, Ramón Ángel, «Análisis de la estructura de la Sociedad Cooperativa de Trabajadores "San Miguelito" S.C.L., para aplicar la administración por objetivos», Tesis de Licenciatura, Universidad de Sonora, 1978, p. 14. http://www.bidi.uson.mx/ TesisIndice.aspx?tesis=4196; Galindo Merchant, Agripina, Las sociedades cooperativas en México: algunas observaciones sobre su funcionamiento, México, Universidad Autónoma Metropolitana Unidad Azcapotzalco, 1987, p. 9.

20 Ibidem, p. 15. http://www.bidi.uson.mx/TesisIndice. aspx?tesis=4196 
En la década de 1870 fueron varias las mutualidades que se transformaron en cooperativas:

— La Asociación Potosina de Obreros en 1871

- La Suprema Hermandad en 1872, en Aguascalientes

- La Fraternidad Jalisciense en Guadalajara en 1874

- Sociedad de Artesanos Católicos de Morelia

- Cooperativa textil poblana

— La mutuocooperativa «El Porvenir» en Veracruz

- Xicoténcatl, Unión de Tablajeros y Comerciantes del Ramo de Carnes en Xochimilco

El 20 de noviembre de 1872 Juan Mata Rivera, en un discurso con motivo del aniversario de la sociedad de rebosería pidió la transformación de las sociedades mutualistas a sociedades cooperativas. ${ }^{21}$ Producto de la propaganda en favor del cooperativismo el 16 de septiembre de 1873 surge en la Ciudad de México el «primer taller cooperativo» dedicado a la sastrería, fue el primer taller que empleo el nombre de cooperativa debido a sus lineamientos y alcances. Así se forma en México la primera cooperativa de producción, no obstante, la división entre sus dirigentes propició el cierre del taller en 1976.

Entre los objetivos de este taller cooperativo estaban:

- Mejorar la situación social y económica de la clase obrera

- Proteger a la clase obrera del capitalismo y de los maestros de talleres

- Formar una gran familia obrera

- Ayudar a los obreros en sus necesidades

- Proteger la producción artesanal

- Formar una red de contactos entre los obreros de los Estados y la capital de la República.

- Hacer consiente a los obreros de sus derechos y obligaciones, respecto a las artes y oficios.

De acuerdo a los objetivos podemos darnos cuenta que, si bien las asociaciones de trabajadores (obreros) ya eran denominadas como cooperativas; en estos momentos más que ser sociedades cooperativas eran una mezcla de mutualismo y cooperativismo, debido al desconocimiento de los principios universales del cooperativismo por parte de los dirigentes. ${ }^{22}$ No obstante, podemos decir que el cooperativismo es una forma de

21 Ibidem, pp. 14-15. http://www. bidi.uson.mx/TesisIndice.aspx?tesis=4196

22 Galindo Merchant, Agripina, Las sociedades cooperativas en México: algunas observaciones sobre su funcionamiento, México, Universidad Autónoma Metropolitana 
asociación integrada por personas con base en intereses comunes y en los principios de solidaridad, esfuerzo propio y ayuda mutua, con el propósito de satisfacer necesidades individuales y colectivas a través de la realización de actividades económicas de producción, distribución y consumo, que surge en el siglo XIX como una alternativa de las clases trabajadoras para enfrentar las condiciones de vida originadas por el capitalismo.

En las sociedades mutualistas los miembros pagaban una cuota mensual que les daba derecho a un salario durante una cierta cantidad de días, cuando estaban enfermos y una ayuda más baja en los días subsecuentes; también residían atención médica gratuita y en caso de fallecimiento los gasto los absorbía la mutualidad.

Las sociedades mutualistas se caracterizaban por estar integradas por artesanos urbanos, la ayuda entre los miembros, se formaba una caja de ahorro para accidentes. Esta forma de organización tenía algunas limitantes que impidieron su desarrollo y permanencia; por ejemplo, los ahorros no se empleaban productivamente, los fondos se restringían de acuerdo a las aportaciones y algunas mutualidades eran clubes políticos. Estos aspectos propiciaron que los integrantes de las mutualidades dudaran de la conveniencia de las mismas y empezaron a perder el interés en ellas para pasar a la formación de cooperativas. ${ }^{23}$

Poco a poco las mutualidades empezaron a verse como desfasadas ante el sistema cooperativista y las cajas de ahorro, que previamente desarrolladas en el extranjero, empiezan a llegar a México. En estas propuestas se nota la influencia de impulsores cooperativistas europeos como Felipe Buchez, Raiffeisen y Schulze. Así, el 18 de agosto de 1876, año en que cerró la primera cooperativa de producción, se forma la primera cooperativa de consumo. Esta sociedad fue llamada oficialmente «Asociación Cooperativa de Consumo de Obreros Colonos» integrada por colonos de la colonia obrera Buenavista; misma que crea la primera tienda o almacén cooperativo. La falta de experiencia, entre otros factores, propiciaron que esta primera cooperativa de consumo funcionara en debida forma; no obstante, llego a tener cientos de afiliados. ${ }^{24}$

Adriana Olvera menciona que las cooperativas unían distintas características: cooperativismo, mutualismo, rasgos de organización gre-

Unidad Azcapotzalco, 1987, p. 10; Méndez Montoya, Ramón Ángel, «Análisis de la estructura de la Sociedad Cooperativa de Trabajadores "San Miguelito" S.C.L., para aplicar la administración por objetivos», Tesis de Licenciatura, Universidad de Sonora, 1978, p. 15. http://www.bidi.uson.mx/TesisIndice. aspx?tesis=4196

23 Olvera López, Adriana, op. cit., p. 34.

24 Méndez Montoya, Ramón Ángel, op. cit., p. 15. http://www.bidi.uson.mx/Tesislndice. aspx?tesis $=4196$ 
mial, funciones incipientes de sindicalismo. No obstante, estas nuevas asociaciones empezaron a plantear características laborales más avanzadas en defensa de los derechos laborales, que no había en las mutualidades. ${ }^{25}$ Así las cooperativas se convirtieron en el mecanismo para evitar la extorción del trabajador por parte del capitalismo, ya que los trabajadores podían ser propietarios del capital invertido y coparticipes de las ganancias generadas. En este sentido, las mutualidades se quedaron rezagadas ante las necesidades obreras. ${ }^{26}$

El movimiento que se produjo en México en la década de 1860 y 1870 en torno a estas nuevas formas de organización, estaba encabezado por maestros de gremios y propietarios de pequeños talleres que fueron paulatinamente remplazados por la industria. Por ello las primeras asociaciones de trabajadores son de carácter gremial y mutualista; además de que un gran número de ellas se ubicaban en el campo, ya que México era una economía eminentemente rural. ${ }^{27}$

De acuerdo a lo expuesto podemos decir, que el cooperativismo fue una alternativa para los campesinos, obreros y artesanos; que ante las desventajas frente al capitalismo encontraron en estas organizaciones una alternativa para solucionar los problemas de producción y consumo. El cooperativismo fue una forma de organización que evolucionó a partir de los gremios y sociedades mutualistas. Así durante las décadas de 1860 y 1870 se dio un crecimiento del movimiento cooperativo, mismo que se verá disminuido durante el Porfiriato; ya que el gobierno de Porfirio Díaz ve a estas asociaciones como un peligro para la estabilidad del régimen debido a la doctrina e ideología a las que estaban sujetas. ${ }^{28}$

\section{El cooperativismo durante el porfiriato}

El régimen de Porfirio Díaz coincide con el ascenso y consolidación de la fase imperialista del capitalismo mundial; por ello uno de los principales objetivos del régimen fue crear las condiciones idóneas para el desarrollo de la economía y la consolidación de los empresarios. La edificación de puertos, el establecimiento de fábricas, el tendido de vías de ferrocarril, la construcción de líneas eléctricas, entre otros aspectos,

\footnotetext{
25 Olvera López, Adriana, op. cit., p. 36.

26 Idem.

27 Olvera López, Adriana, op. cit., p. 30-31.

28 Galindo Merchant, Agripina, op. cit., pp. 10-11.
} 
eran vistos como los elementos esenciales para el desarrollo del país. ${ }^{29}$ Para poder realizar lo anterior se expidieron políticas nacionales y estatales en favor del desarrollo económico e industrial, como consecuencia surgieron diversas industrias: vidriera, cervecera, jabonera, tabaquera, cementera, ferroviaria, fundidora; no obstante, la producción individual y local logró subsistir. De esta forma, la inversión creció y con ella el mercado nacional, así la población tuvo acceso más fácil a los productos, pero con la aceleración de los procesos capitalistas los campesinos, artesanos y obreros se vieron perjudicados y empobrecidos, ante la imposibilidad de competir con los grandes empresarios.

Frente al crecimiento comercial, el gobierno de Porfirio Díaz apostó a la intervención del Estado en la economía, aprovechando con ello el auge de las exportaciones y la inversión extranjera. En este contexto, en los primeros años del régimen porfirista la propaganda sobre las cooperativas que se realizó en la década de 1870 continuó haciéndose en distintos periódicos obreros; por ejemplo, en El hijo del trabajo, La abeja poblana, El Socialista, así como en otros periódicos de circulación nacional. Una de las ideas más difundidas en estos periódicos era la de que los recursos económicos no deberían estar estancados o dárseles solamente a los enfermos, sino que debería ser invertido y distribuido entre todos los miembros integrantes de la cooperativa.

El ideal era crear una sociedad de pequeños productores libres e independientes del capital. Esta propaganda se prolongó hasta 1890, produciéndose con ello un primer ciclo de cooperativismo mexicano, que tuvo principal relevancia en los sectores de ahorro y crédito, agrícola y de servicios en general. ${ }^{30}$

El cooperativismo durante el régimen porfirista se puede dividir en dos etapas, la de la paz porfiriana (1877-1888) y la del reconocimiento jurídico de las cooperativas como entidad asociativa y empresarial que se caracterizaba por su incorporación a los procesos de modernización económica impulsados durante el Porfiriato. ${ }^{31}$

En la primera etapa surgieron cooperativas de producción, crédito, consumo y ahorro, mediante las cuales se pretendía que los trabaja-

29 Guerra, Francois Xavier, México. Del antiguo régimen a la Revolución, México, Fondo de Cultura Económica, 1995, 303-314.

30 Rojas Herrera, Juan José, «La relación del Estado y las cooperativas durante el Porfiriato: 1876-1911», IX Congreso Internacional Rulescoop, 2015, p. 4. Recuperado de http://sedici.unlp.edu.ar/bitstream/handle/10915/50616/Documento_completo. pdf? sequence=1 (fecha de consulta: 27 de septiembre de 2020).

31 Rojas Herrera, Juan José, op. cit., p, 2. Recuperado de http://sedici.unlp.edu.ar/bitstream/handle/10915/50616/Documento_completo.pdf?sequence=1 (fecha de consulta: 27 de septiembre de 2020). 
dores tuvieron mejores condiciones de vida. En noviembre de 1877 un grupo de veinte personas encabezadas por Francisco de P. González fundaron la cooperativa del "Banco Social del Trabajo», que posteriormente se denominaría «Sociedad Democrática, Industrial y Capitalista». Las bases provisionales de la sociedad incluían algunas cláusulas de carácter mutual y cooperativo, siendo importante señalar que la sociedad adoptó por lema: «Uno para todos y todos para uno».

El Banco Social del Trabajo se conformaría como una sociedad mutualista, con el objeto de que la clase trabajadora se protegiera mutuamente y proporcionar empleo al trabajador en el arte o industria que ejerciera. La admisión quedaría restringida a quienes practicaran un arte o industria, sin distinción de nacionalidad, sexo, ni edad. Al momento de ingresar a la sociedad, los socios debían pagar cada semana, la cantidad de doce y medio centavos, cuyos recursos se utilizarían tanto para hacer préstamos a los socios, como para crear periódicamente talleres. ${ }^{32}$

Además del Banco Social del Trabajo, en 1882 se creó el «Banco Popular de Obreros» y en 1883 el Banco de Empleados. Estos bancos plantearon el crédito popular como un medio de ayuda y organización de las clases subalternas.

Esta nueva forma de asociación (cooperativas) trajo distintas aportaciones, una de ellas fue el poder disponer del fondo reunido por los socios para invertirlo en la producción, cosa que no se hacía en las mutualidades; en éstas, al contrario, el dinero era un fondo estático y si las condiciones de los miembros eran adversas el fondo de ayuda era insuficiente, lo que originaba problemas porque no todos los miembros recibían los servicios de ayuda. En cambio, con las cooperativas el dinero les permitía a los socios obtener un rendimiento, que los hacía participes y usufructuarios de las ganancias de la cooperativa. ${ }^{33}$ A pesar de que las cooperativas fueron innovadoras, no fue posible que todos los obreros o artesanos pudieran constituirlas debido a la falta de recursos; en este sentido, autores como Woldenberg y Leal consideran que el movimiento cooperativo fue algo elitista porque permitía mayores beneficios a aquellos trabajadores de renombre. En este sentido, la idea de cooperativa fue retomada por la burguesía, y no tanto por el proletariado.

La política gubernamental implementada durante el Porfiriato se caracterizó por favorecer las grandes industrias y no dar cabida a las

32 Juan José Rojas Herrera, op. cit., p. 4-5.

33 Olvera López, Adriana, op. cit., p. 35. 
organizaciones sociales que surgieron como consecuencia de las mismas; al contrario, se buscó mantener al obrero en la ignorancia y el sometimiento. ${ }^{34}$ En este sentido, solo subsistieron las sociedades mutuales y cooperativas encabezadas por gente estrechamente relacionada con el gobierno o que aceptaron la tutela del Estado. ${ }^{35}$ Por ejemplo, en septiembre de 1879 con la participación de destacadas personalidades: industriales, políticos, intelectuales y profesionistas se creó la Caja Popular Mexicana, su presidente era Vicente Riva Palacio. Se suponía que la participación de destacadas personalidades redundaría en un mejor trabajo que los obreros y artesanos para llevar a buen destino la cooperativa, pero esto no fue así. Según Rojas Coria el fracaso de esta cooperativa se debió a la falta de confianza de los artesanos, obreros y pequeños comerciantes e industriales y debido a la presión del capitalismo que avanzaba en todas las áreas de la actividad económica. ${ }^{36}$

A pesar del desarrollo industrial y comercial, aún había necesidades que solo podían ser satisfechos por medio de productos regionales. Esta situación propició la organización de pequeños productores y consumidores locales. ${ }^{37}$ En el espacio rural el gobierno porfirista implementó las leyes liberales de desamortización de las propiedades comunales de los pueblos, a través de las cuales muchos campesinos indígenas perdieron sus tierras. También se deslindaron tierras baldías, y se proyectó la colonización de tierras. Ambas situaciones originaron un insipiente desarrollo de la clase proletaria agrícola, la cual tuvo que vender su fuerza de trabajo para poder sobrevivir. En este contexto, en el campo mexicano también empezaron a aparecer asociaciones para hacer frente al capitalismo; por ejemplo, en 1886 se creó la colonia «Porfirio Díaz» en Tlalpixalco, Estado de México, cuyo fin principal fue resolver necesidades colectivas de obreros desempleados de la Fábrica de Hilados y Tejidos de Contreras. ${ }^{38}$

A partir de la década de 1830 se instalaron fábricas textiles en el valle de México, que empezaron a trabajar con maquinaria y trabajadores extranjeros; entre las cuales se encontraban La Magdalena, San Ildefonso y Miraflores. ${ }^{39}$ Las condiciones de trabajo de los obreros eran

34 Olvera López, Adriana, op. cit., p. 33.

35 Galindo Merchant, Agripina, op. cit., p. 10-11.

36 Rojas Herrera, Juan José, op. cit., p, 5-7. Recuperado de http://sedici.unlp.edu.ar/bitstream/handle/10915/50616/Documento_completo.pdf?sequence=1 (fecha de consulta: 27 de septiembre de 2020).

37 Olvera López, Adriana, op. cit., p. 32-33.

38 Ibidem, p. 40.

39 Trijillo Bolio, Mario, Operarios fabriles en el Valle de México, 1864-1884, México, CIESAS, El Colegio de México, 1997a. 
precarias y la estabilidad laboral dependía de conservar el trabajo pese a las reducciones de salarios. El cierre de una fábrica obligaba a los trabajadores a emigrar a otras poblaciones en busca de trabajo, para paliar las contingencias los trabajadores se unían en sindicatos y mutualidades; así, en 1865 los trabajadores de las fábricas de San Ildefonso y La Colmena formaron la sociedad mutua del Ramo de Hilos y Tejidos del Valle de México para poder mejorar las condiciones laborales. ${ }^{40}$

En 1830 La Magdalena fue fundada como un pequeño obraje, para 1850 ya era una de las fábricas más grandes del valle de México con una producción de 54,870 mantas de algodón y 520,147 piezas de hilaza. ${ }^{41}$ En 1879 el dueño de La Magdalena realizo cambios tecnológicos al sustituir la maquinaria por telares modernos, lo que obligo a un reacomodo en las fuerzas de trabajo y redujo el número de obreros; ${ }^{42}$ para 1886 los obreros de la magdalena iniciaron una huelga que culminó en el despido de varios trabajadores. Los grupos socialistas pelearon por reubicar a los trabajadores, presionando al gobierno de Porfirio Díaz para que no los desamparara, y éste consideró formar una colonia agrícola-industrial con los obreros despedidos. Así inicio el proyecto de formar una colonia de obreros dedicada a la producción de seda en terrenos de la hacienda de Tlapizalco, en el distrito de Tenancingo. ${ }^{43}$

El caso de la colonia-agrícola de Tlapizalco es ilustrativo de los estragos que el desarrollo empresarial originó en los trabajadores y del interés de los trabajadores por formar asociaciones para hacer frente a los estragos originados por el desarrollo de la industria. Las condiciones de trabajo de los trabajadores de la fábrica de La Magdalena también fueron enfrentadas por obreros de distintas fábricas del valle de México y otras regiones del país.

La segunda etapa de las cooperativas durante el régimen porfirista se caracteriza por la regulación de las cooperativas a través de un marco legal y la incorporación de las mismas al mercado. Como parte del nuevo marco legal para regir y controlar las cooperativas en 1889 fueron reconocidas legalmente en el Código de Comercio, adquiriendo con ello personalidad jurídica, conmiserándolas dentro de las sociedades mercantiles. Iniciándose así un proceso para legalizar su existen-

40 Ibidem, pp. 127, 171-176.

41 Gracia Luna, Margarita, Los orígenes de la industria en el Estado de México (1830-1930), Toluca, Gobierno del Estado de México, 1998, p. 29.

42 Trujillo Bolio, Mario, «La fábrica La Magdalena Contreras (1839-1910). Una empresa textil precursora en el valle de México», en Carlos Marichal y Mario Cerutti (comps.), Historia de las grandes empresas en México, 1850-1930, México, FCE, 1997b, p. 265.

43 Villalobos, Liborio, Las obreras en el porfiriato, México, Plaza y Valdés, 2002, p. 312. 
cia. De esta forma el Estado, como promotor del desarrollo, comienza a intervenir en el fomento de sociedades cooperativas considerándolas como pequeñas empresas en las cuales puede ejercer control mediante las disposiciones legales establecidas. ${ }^{4}$

Poco apoco, las cooperativas fueron adquiriendo cierto orden, lo que las llevo a sobrevivir al movimiento revolucionario iniciado en 1910. En 1917 las sociedades cooperativas adquieren rango constitucional al ser incluidas en los artículos 28 y 123 de la Constitución Política del mismo año. En ella se les considera como organizaciones de la clase trabajadora que tienen como objeto principal la defensa común de los intereses de ésta. ${ }^{45}$

En 1927 Plutarco Elías Calles dictó la primera ley sobre cooperativas, misma que fue concebida con la idea de desarrollar el cooperativismo ideado por Raiffesen, líder cooperativista alemán a mediados del siglo XIX. En 1933 fue aprobada la segunda ley de cooperativas, esta ley incluyó a los socios y los rendimientos, lo que los diferenció de las sociedades mercantiles, donde había accionistas y utilidades. Cinco años después fue publicada la primera Ley General de Sociedades Cooperativas de $1938 .{ }^{46}$

\section{Ley general de sociedades cooperativas del 15 de febrero de 1938}

Esta ley no sufrió modificaciones durante muchos años, hasta que el 3 de agosto de 1994 se publicó la nueva Ley General de Sociedades Cooperativas, que actualmente está en vigor. La ley de 1838 dividió a las sociedades cooperativas en dos tipos:

1. Cooperativas de Producción. Son aquellas integradas por individuos de la clase trabajadora que aportan a la sociedad su trabajo.

2. Cooperativas de Consumo. Son aquellas en las que los socios se aprovisionan a través de ella o utilizan sus servicios.

En general, la estructura de las sociedades corporativas era la misma; pero con algunas acepciones. Por ejemplo, en una sociedad cooperativa de producción existe un comisionado de la organización de la producción, en tanto que en una de consumo no; aunque, existe

\footnotetext{
44 Galindo Merchant, Agripina, op. cit., p. 10-11.

45 Galindo Merchant, Agripina, op. cit., p. 11.

46 Ibidem, p. 13.
} 
un comisionado de la organización de la distribución. Además, en una cooperativa de producción existe una comisión de control técnico y en la cooperativa de consumo no existe. ${ }^{47}$

Toda sociedad cooperativa tiene una estructura basada en tres aspectos fundamentales dirección, administración y vigilancia, que están compuestos por órganos principales: asamblea general, consejo de administración, consejo de vigilancia y las comisiones señaladas en la ley. Es decir, toda sociedad cooperativa debe de tener una estructura. ${ }^{48}$

En los años treinta del siglo anterior se fundaron las cooperativas más grandes, como la productora de Cemento Cruz Azul, la del periódico Excélsior y la del periódico La Prensa. ${ }^{49}$

\section{Cooperativismo en México después del cardenismo}

Tal como se ha mencionado, puede decirse que el sexenio del presidente Lázaro Cárdenas (1934-1940) comprende un periodo de ascenso para el movimiento cooperativo mexicano, el gobierno cardenista propició la constitución de cooperativas, tanto en el campo como en la ciudad. ${ }^{50}$

En ese entonces el cooperativismo se concibió como un sistema apropiado para organizar empresas productivas y además promover socialmente a contingentes de trabajadores, con el objetivo de ampliar y fortalecer al movimiento cooperativo, esto motivó al cardenismo para renovar al cooperativismo mexicano sobre bases más firmes, populares y solidarias con los sectores obreros y campesinos. ${ }^{51}$

A palabras de Cárdenas, dos causas principales propiciaron efectos positivos en la economía del país

«...la entrada de capitales repatriados, o bien de extranjeros que aprovechaban la política de libre cambio o buscaban refugio en el país ante los problemas de la guerra,...Al crecer el ingreso nacional

47 Méndez Montoya, Ramón Ángel, op. cit., p. 17. http://www.bidi.uson.mx/TesisIndice. aspx?tesis $=4196$

48 Ibidem, p. 18-20. http://www.bidi.uson.mx/TesisIndice.aspx?tesis=4196

49 Fritz-Krockow, Bernardo, "Evolución del cooperativismo mexicano», Comercio Exterior, vol. 36, núm. 9, 1986.

50 Labriega Villanueva, Pedro Alfonso. Citado en Izquierdo, M. Comentarios entorno a las sociedades cooperativas en México. Universidad de Deusto página 56

51 ldem. 
en los Estados Unidos por su preparación para entrar al conflicto bélico, la demanda de ciertos bienes y servicios estimuló su producción en México para satisfacer el mercado norteamericano». ${ }^{52}$

De manera interna el mercado también se vio favorecido por el crecimiento poblacional, pues pasó de 19 a 25 millones de personas de 1940 a 1950; de igual modo la urbanización del país sufrió cambios notorios, pese a que la población era mayoritariamente rural, veinte años después, de 1940 a 1960 la población urbana paso de ser un $35 \%$ a un $50 \%$ igualando a la población rural. 53

Ante este panorama, la población industrial cobraba cada vez mayor importancia, por ello la década de 1940 se convierte también en la década de los pactos, mismos que se encaminaban a hacer de la explotación y la alianza entre la clase trabajadora y la burguesía, el sustento del crecimiento industrial y económico del país. ${ }^{54}$

En el año de 1941 se creó la Secretaria de Trabajo y Previsión Social, así como los preparativos para la fundación del Seguro Social, lo que le permitiría al Estado participar más profundamente en los asuntos laborales. Así mismo, el Estado auspició la creación del Consejo Nacional Obrero, así como el Consejo Nacional Patronal y el Consejo Superior de Defensa.

En sus inicios estas políticas lograron persuadir a los trabajadores de evitar cualquier expresión de descontento que pudiera propiciar el desequilibrio del país; pues lo que se necesitaba en ese entonces era que todos los sectores apoyaran la construcción de una nación fuerte, con una economía sólida como para poder independizarse del exterior. Los trabajadores no querían perturbar un desarrollo prometedor $y$, a pesar de todo el sacrificio que se les estaba imponiendo, podía pensarse que no era tan severo si la expansión industrial creaba nuevos empleos y se tenía una visión nacionalista. Así, en este mismo año se creó el Pacto de Unidad Obrera, que proporcionaba su apoyo al país por la vía de la no huelga. ${ }^{55}$

«El Pacto de la Unidad Obrera Nacional, que todas las centrales de trabajadores patrióticamente suscribieron, a invitación del Ejecutivo, son efecto de eliminar las luchas intergremiales, vigorizar la democracia sindical y someter voluntariamente a la conciliación y al arbitraje la

52 Cárdenas, Enrique. La hacienda pública y la política económica, 1929-1958, Fondo de Cultura Económica, México, 1994, pp. 92-93

53 Olvera López, Adriana. Op. cit., p

54 Idem

55 Ibidem, p. 111 
solución de los conflictos, a efecto de cooperar eficazmente en el esfuerzo que el estado de guerra impone al país. De este pacto se deriva un aumento de eficacia y disciplina en los centros de trabajo, un estímulo para modernizar los equipos y facilidades para transformar las industrias y contribuir a resolver el problema de los desocupados» ${ }^{56}$

En este contexto surge otro pacto igualmente importante para el movimiento obrero, el Pacto Obrero Industrial, cuya «idea central era garantizar una unión de clases con el objetivo de seguir impulsando el desarrollo económico del país, conseguir una economía autónoma y evaluar el nivel de vida de las masas del pueblo mexicano». ${ }^{57}$

También, durante la administración del presidente Manuel Ávila Camacho (1940-1946), se creó el Banco Nacional de Fomento Cooperativo con el propósito de corregir las limitaciones que tenía el Banco Nacional Obrero del Fomento Industrial, cuyo margen de acción era muy estrecho y no tenía capacidad financiera para auxiliar con el crédito a los organismos con los que debía operar (cooperativas y grupos de trabajadores), este nuevo banco también se encargó de organizar y administrar un departamento de ahorro obrero. ${ }^{58}$

«Se ha impulsado el movimiento cooperativo de los trabajadores, habiéndose alentado la fundación de una cooperativa única de trabajadores del Estado y el establecimiento de federaciones que se asociarán a una Confederación Nacional Cooperativa». ${ }^{59}$

Se acordó también que todos los permisionarios que exploten servicios de autotransporte en los caminos nacionales o particulares de concesión Federal contarían con un plazo de 365 días, para constituirse en sociedades cooperativas. Regulación que también se hizo sentir en las cooperativas pesqueras, a través del reglamento en materia de cooperativas federales de pescadores, el cual señalaba que «las aduanas marítimas y fronterizas no permitirían la exportación de pescado procedente de las sociedades cooperativas, si no es con la autorización de la federación a que estas pertenezcan». ${ }^{60}$ Obligando a la organización de

56 Congreso, Cámara de diputados, citado en Olvera López, Adriana. p.111

57 Reyna, J. y Miquet, citado en Olvera López, Adriana. p. 112

58 Bautista, A. El desarrollo del cooperativismo como parte del sector social de la economía p. 215 https://goo.gl/P7gZY9

59 Congreso, Cámara de diputados, citado en Olvera López, Adriana. p.112

60 Diario Oficial de la Federación, Reglamento de los artículos 73, fracción III, y 82 de la Ley General de Sociedades Cooperativas en materia de Cooperativas Federales de Pescadores, 15 de octubre de 1941 
Federaciones de cooperativas para llevar un control más estricto de la producción y funcionamiento de este tipo de organizaciones. ${ }^{61}$

Hugo Rangel Couto señala que «Hace falta la ayuda económica,..., pero no como dádiva, sino como crédito recuperable, que solo debe otorgarse cuando haya perspectivas de éxito que se adviertan de un estudio cuidadoso de viabilidad económica y se les dé luego asesoría técnica eficiente». ${ }^{62}$

Como se menciona, estos elementos eran imprescindibles para lograr el éxito de las cooperativas recién creadas, un correcto seguimiento a su situación financiera era garantía de funcionamiento y no de quiebra de estas sociedades, lo cual permitiría un aprovechamiento mayor de los recursos. Aun así, a partir de 1943 comienzan a aparecer decretos y acuerdos de disolución de cooperativas. Las razones principales para disolver las sociedades cooperativas eran dos: que no hayan iniciado o continuado sus actividades de acuerdo con las bases constitutivas de la cooperativa, o bien que se hayan liquidado sin hacerlo de acuerdo con el artículo 24 de la Ley General de Sociedades Cooperativas, haciéndose acreedores a las sanciones estipuladas en el art. 46 de la misma Ley. ${ }^{63}$

Tal fue el caso de la Sociedad Cooperativa de Construcción y Consumo de servicios «Salvador Díaz Mirón» (30 de noviembre de 1943), la Sociedad Cooperativa Ejidal de Venta en Común Chapultepec (02 de diciembre de 1943), Sociedad Cooperativa de Trabajadores de Transporte de Carga Monterrey-Saltillo-Torreón (14 de diciembre de 1944), Sociedad Cooperativa de Electricidad y Hielo de Progreso (27 de septiembre de 1950), entre muchas otras, que son las sociedades con las que se inició el retroceso del movimiento, al existir esta masiva cancelación y revocación de autorizaciones para funcionar que efectuó el gobierno a partir de entonces.

Por otra parte, Una vez que terminó la Segunda Guerra Mundial, derivado del apoyo, basado en el nacionalismo que el Estado exigía al movimiento obrero, los trabajadores decidieron abandonar el pacto de no huelgas, entre otras razones, porque su nivel de vida empeoraba notablemente, pues la tasa de crecimiento del costo de la vida obrera era superior al crecimiento de los salarios, en mayor parte ocasionado por la posguerra. ${ }^{64}$

\footnotetext{
61 Olvera López Adriana. Op. cit.,p. 12-13

62 Idem.

63 ldem.

64 Ibidem, p. 115
} 
Los trabajadores se encontraban descontentos con el Estado, pues el movimiento obrero no contaba con medios eficaces de expresión que no llevaran una represalia inmediata. Así, en 1945 la CTM rompe el compromiso de no hacer huelgas, pues la Segunda Guerra Mundial había concluido. Lombardo dirigía una de las corrientes más importantes: el nacionalismo económico, el cual pretendía la autosuficiencia del país, la sustitución de importaciones, la hostilidad al capital extranjero y la independencia económica, así como la intervención del Estado y el regreso a los planteamientos cardenistas.

Corriente que presentaba intereses contrarios a los del liberalismo económico, principal teoría manejada por los gobiernos de Ávila Camacho y Alemán. Quienes buscaban restringir la inversión estatal y ampliar la participación extranjera en las inversiones. ${ }^{65}$

El gobierno dejó de buscar la coincidencia con la principal central del movimiento obrero cuando Lombardo, que encabezaba la CTM, decidió formar un nuevo partido político, el Partido Popular. Ante esto el gobierno tuvo que enfrentarse a la disyuntiva de atraer la central obrera al seno del partido, desaparecerla, o dejarla vivir como un ente opositor.

Cuando las dos últimas opciones representaban poca ventaja al gobierno, tuvo que decidir por la primera, ya que la conformación del partido único exigía bases populares, de las que los obreros formaban parte esencial. Alemán logró cooptar la central, pero tuvo que atravesar por conflictos con importantes sindicatos. ${ }^{66}$

En este punto, es importante señalar que el sector agrario sufrió una transformación radical en estos años. La propiedad de la tierra va del ejido colectivo cardenista a la pequeña gran propiedad como base del desarrollo agrícola del país.

El movimiento agrario después de su separación del movimiento obrero, ocurrida durante el gobierno de Cárdenas, no volvería a experimentar un desarrollo independiente del Estado y mucho menos se constituiría en una fuerza antagónica de las políticas estatales, por lo menos en la siguiente década.

Los campesinos también tenían la esperanza de volver a los tiempos cardenistas del reparto agrario; esperanza que poco a poco se veía defraudada al llevarse a cabo las políticas de Ávila Camacho. La mayor intervención del Estado en todos los procesos (tanto productivos como distributivos) del agro condujo a que este sector económico quedara reducido a la dependencia absoluta de la paternidad gubernamental. ${ }^{67}$

65 Idem
66 Ibidem, p. 18
67 Ibidem, p. 17 
El movimiento agrario dejo de tener importancia como fuerza opositora, mientras que el movimiento obrero ante la disolución del pacto, comenzó a sufrir las represalias que tuvo contra el Estado, pues éste se dio a la tarea de debilitar la principal central de trabajadores.

El sucesor de Ávila Camacho, Miguel Alemán encabezó un gobierno caracterizado por la enorme desigualdad de la distribución del ingreso, heredado por el gobierno anterior. Así, las nuevas relaciones tuvieron como principal marco las modificaciones en materia agraria, así como el ajuste del movimiento obrero a las nuevas condiciones capitalistas industriales. ${ }^{68}$

Después de que la CTM rompiera con Lombardo en 1948, este creo la alianza de obreros y campesinos, como una central rival a aquella, y estaba compuesta por la Central Única de Trabajadores (CUT), la cual, a su vez estaba compuesta por sindicatos muy fuertes, como el ferrocarrilero o el petrolero, de esta forma se funda el Partido Popular en junio de 1948, teniendo como eje central la Alianza de Obreros y Campesinos, la cual cambia su nombre a Unión General de Obreros y Campesinos de México (UGOCM).

Ante esto, el gobierno le negó a la UGOCM, la capacidad de representar legalmente a sus afiliados, de esta forma pocos trabajadores decidieron quedarse a formar parte de la UGOCM, pues sus demandas no podían ser atendidas, ni contaban con representación legal suficiente para hacer valer sus derechos. Así que los obreros decidieron volver a la CTM.

Hacia 1949 Lombardo había sido derrotado, del mismo modo que su Partido Popular. Con el gobierno de Alemán, el país sufre el tránsito de la Segunda Guerra Mundial, y su posguerra, la cual cerró posibilidades de exportaciones, así como la acumulación de divisas para respaldar la moneda nacional.

El grave contexto externo de la guerra puso en riesgo la economía nacional, comenzaron a verse consecuencias sobre la moneda y el comercio exterior. Las huelgas y demás movilizaciones obreras no se hicieron esperar al reclamar el ajuste al salario, llevando a varios grupos obreros a demandar el control de las empresas ante esta crisis, sin embargo, el Estado parecía no perder la situación de control, otorgando facilidades de inversión y protección a las empresas. De este modo se permitió la formación de grandes fortunas a inversionistas industriales. ${ }^{69}$

68 Ibidem, p.18

69 Ibidem, pp.19-21 
Al respecto, Rojas Coria explica:

«... no se le puede atribuir nada significativo en favor del cooperativismo, excepto que unificó las distintas leyes y decretos que fueron expedidos por presidentes anteriores para proteger a las sociedades cooperativas pesqueras, en una sola Ley General de Pesca, en la que se dedicaron siete especies marinas (las más comerciales) para ser capturadas exclusivamente por sociedades cooperativas pesqueras» ${ }^{70}$

Así vemos como surgieron cooperativas pesqueras y subsidios para las mismas, pero pareciera que fue a costa de otras cooperativas, ya que de 1950 a 1952, nuevamente hubo una gran liquidación de sociedades cooperativas de todo tipo, principalmente de consumo, como la Sociedad Cooperativa de Consumo, Guadalupe Victoria (18 de agosto de 1951), o la Sociedad Cooperativa de Productores de Sombreros de Palma en Colima (30 de agosto de 1952). ${ }^{71}$

A partir de este gobierno la caída del cooperativismo es clara, pues no se volverá a considerar como parte de la política social que mantenía bajo control a las masas, ya que estas ya se encontraban controladas.

Para la siguiente administración, que fue la de Adolfo Ruiz Cortines (1952-1958), se impuso una política de austeridad para tratar de controlar el desequilibrio en las finanzas. Aun así, se abocó a proporcionar beneficios a las sociedades cooperativas, al conceder la extensión de impuestos sobre la renta y sobre los ingresos mercantiles a las cooperativas de producción y consumo.

Este nuevo gobierno se dio a la tarea de unificar todos aquellos sindicatos que se encontraban dispersos en una sola central llamada CROC, que quedó inserta en el PRI en 1952, controlando así el movimiento sindicalista hasta entonces independiente.

Mientras tanto, el contexto económico empezaba a gozar del llamado desarrollo estabilizado, sin embargo, la desigualdad social fue en aumento. Tras el velo de una economía que avanzaba firmemente en la exitosa ruta industrial, se ocultaba el descontento social.

Este crecimiento del Estado ya no requería de mayores medidas de control, excepto por el solo hecho de gobernar de forma autoritaria y antidemocrática. Así el Estado comenzó a desligarse del movimiento cooperativista y del apoyo que brindaba a este. Comenzaron a revo-

70 Coria, R. citado en Olvera López, Adriana, p.121

71 Diario Oficial de la Federación, Acuerdos y Decretos que revocan la autorización otorgada a las cooperativas para funcionar, varios años. Citado en Olvera López Adriana, p.122 
carse las autorizaciones para funcionar, incluso a cooperativas pesqueras $^{72}$, las de transporte y consumo sindicales en toda la República.

Poco a poco se le va abandonando como medio de organización eficaz, como clase, los obreros no lograban unificarse del todo, y el Estado absorbía cualquier organización propia del movimiento, lo que no ocurrió con el brote obrero en 1958 y 1959, cuando ante el bajo nivel de vida y poder adquisitivo, así como el decreciente desempleo, los trabajadores se rebelaron ante el charrismo de sus sindicatos, lo cual se conjugó con los tiempos electorales en que Adolfo López Mateos se postulaba como candidato a la presidencia. ${ }^{73}$

A partir de los años sesenta las cooperativas no volverían a formar parte importante de la política gubernamental, existen algunos ejemplos como: La Cooperativa Manufacturera de Cemento «Portland» «La Cruz Azul» (surge alrededor de 1931, cuando después de un conflicto obreropatronal la empresa quedó en manos de los trabajadores. ${ }^{74}$ La Cooperativa de Autotransportes «Excelsior» Jalapa-Coatepec-Xico (organizada en 1935); «Editora de Periódicos» (constituida el 10 de junio de 1935); Sociedad Cooperativa de Compras en Común «La Proveedora de la Industria Automotriz» (15 de junio de 1949); Sociedad Cooperativa de Consumo «Tranvías y Luz de México» (alrededor de 1927), entre muchas otras.

De esta lista, 50 años después no sobrevive casi ninguna de las organizaciones, entre otras razones por el casi nulo interés gubernamental por promoverlas «hace falta mayor voluntad política del Estado para motivar a quienes quieren agruparse en este tipo de organización». ${ }^{75}$

Como se ha explicado, México siguió presentando ritmos acelerados de crecimiento del movimiento cooperativo nacional, dentro de los cuales, a nivel nacional durante el periodo de 1938 a 1976 se integraron un total de 6 mil 610 cooperativas, donde 4 mil 298 eran de producción y 2 mil 312 de consumo, sumando un total de 518 mil 596 socios según datos de la Dirección General de Fomento Cooperativo de la Secretaría del Trabajo y Previsión Social. ${ }^{76}$

72 Diario Oficial de la Federación, Acuerdos que revocan la autorización para funcionar a las cooperativas pesqueras de Baja California, 28 de agosto de 1956. Citado en Olvera López, Adriana. p.123.

73 Olvera López, Adriana, pp.121-124

74 Rodríguez Rosa, Antonio. La Revolución sin sangre (El Cooperativismo), citado en Olvera López, Adriana. P.24

75 Periódico El Día, 10 de abril de 2000, entrevista a Guillermo Álvarez Cuevas, presidente de la Sociedad Cooperativa «La Cruz Azul», primera plana. Citado en Olvera López, Adriana, p.25

76 Exposición de motivos de la Iniciativa que expide la ley general de sociedades cooperativas, suscrita por los diputados del grupo parlamentario de Morena y sin Par- 
En ese mismo año surgieron una serie de políticas públicas destinadas al sector cooperativo, se creó la Comisión Intersecretarial de Fomento Cooperativo, cuyo principal objetivo sería apoyar el crecimiento del cooperativismo en México, sin embargo, se entiende que el movimiento cooperativo dependía directamente del Estado, el cual aparentemente se encargó de frenarlo poco a poco, por eso en los años siguientes a 1978 ese crecimiento era muy inferior comparado con los años anteriores, aunque no por ello dejó de colaborar en el desarrollo nacional. Diez años después se volvió a realizar un estudio por parte de la Unidad Coordinadora de Políticas, Estudios y Estadísticas del Trabajo, de la Secretaría del Trabajo y Previsión Social, el cual muestra que para el año 1988 existían un total de 8,017 cooperativas, con un total de 349 mil 47 socios. ${ }^{77}$

Es posible que una de las principales limitantes que les imponen las leyes y los programas de fomento sea el que: «... ven el cooperativismo como un estorbo y no como otra alternativa viable. Muchos funcionarios, la iniciativa privada y no pocos administradores y gerentes nos interpretan como un sistema teóricamente hermoso, pero en la práctica como una utopía». ${ }^{78}$

En este contexto es que el movimiento cooperativo nacional exigía una nueva legislación acorde a sus necesidades y las problemáticas a las que se enfrentaban. Por ello, el 03 de agosto del año 1994 se publicó una nueva ley relativa a este sector; dicha ley proponía adecuar el contenido a las necesidades impuestas por la sociedad, con base en la Constitución Política de los Estados Unidos Mexicanos. Esta ley decretó la desaparición de la Dirección General de Fomento Cooperativo de la Secretaría del Trabajo y Previsión Social, delegando así, la responsabilidad de función y control estadístico del cooperativismo a la Secretaria de Desarrollo Social. ${ }^{79}$

\section{Conclusiones}

Concluimos que en México no se ha podido formar y construir una política gubernamental y una cultura que fortalezca al cooperativismo, el sistema neoliberal y capitalista que existe en nuestro país, no favorece el desarrollo de esta importante institución jurídica y social.

tido. Antecedentes legales. Sistema de información legislativa de la secretaria de gobernación.

77 Ibid.

78 Periódico El Día, Primer Manifiesto Cooperativista, 22 de noviembre de 1999, p. 19 citado en Olvera López, Adriana, p.26

79 ldem. 
La economía solidaria compuesta de organizaciones populares colectivas que buscan resolver las necesidades materiales mediante relaciones sociales, donde la igualdad, la reciprocidad, el sentido de la comunidad, la autoridad colectiva y la sustentabilidad cobran especial importancia, pero que se confrontan con un complejo conjunto de leyes que conforman nuestro marco jurídico mexicano, donde limitan a las sociedades cooperativas, evitando su desarrollo.

Consideramos que las cooperativas en México, por su forma de operar y su estructura administrativa, se han prestado a que sus dirigentes y administradores no estén educados y documentados acerca de la filosofía social de este movimiento, por tanto, se han aprovechado en lo económico y en lo político, circunstancia que ha provocado que no exista un desarrollo y fortalecimiento de un número más de cooperativas en nuestro país.

\section{Bibliografía}

Centro de estudios soCIAles y de opinIón PúbliCA, "Cooperativismo en México», Cámara de Diputados, 2016, p. 5.

FrITZ-Krockow, Bernardo, «Evolución del cooperativismo mexicano», Comercio Exterior, México, vol. 36, núm. 9, 1986, pp. 790-796.

Galindo MerCHANT, Agripina, Las sociedades cooperativas en México: algunas observaciones sobre su funcionamiento, México, Universidad Autónoma Metropolitana Unidad Azcapotzalco, 1987.

Gracia Luna, Margarita, Los orígenes de la industria en el Estado de México (1830-1930), Toluca, Gobierno del Estado de México, 1998.

Gobierno de México, Constitución Federal de los Estados Unidos Mexicanos de 1857, México, Imprenta de F. Guzmán y Hermanos, 1875.

GuerRa, Francois Xavier, México. Del antiguo régimen a la Revolución, México, Fondo de Cultura Económica, 1995.

Inostroza Fernandez, Luis, Movimiento cooperativista internacional. Cooperativismo y sector social en México. México, Universidad Autónoma metropolitana, 1989.

IZquIERDO, Martha E. Comentarios en torno a las sociedades cooperativas en México, Boletín de la asociación internacional de derecho cooperativo. Universidad de Deusto. http://baidc.revistas.deusto.es/article/view/128/236

MÉndez Montoya, Ramón Ángel, "Análisis de la estructura de la Sociedad Cooperativa de Trabajadores "San Miguelito" S.C.L., para aplicar la administración por objetivos», Tesis de Licenciatura, Universidad de Sonora, 1978, p. 14. http://www.bidi.uson.mx/TesisIndice. aspx?tesis=4196;

OlverA LÓPEZ, Adriana, «El Sistema Cooperativo Industrial Mexicano: una revisión histórica 1929-1958», Tesis de Licenciatura en Economía, México, UNAM, 2001. 
Rojas HeRRERA, Juan José, "La relación del Estado y las cooperativas durante el Porfiriato: 1876-1911», IX Congreso Internacional Rulescoop, 2015, p. 4. http://sedici.unlp.edu.ar/bitstream/handle/10915/50616/Documento_completo.pdf?sequence $=1$

SÁNCHEZ SANTIRÓ, Ernest, «El desempeño de la economía mexicana, 1810-1860: de la colonia al Estado-Nación», en Sandra Kuntz Ficker (coorda.), Historia económica general de México. De la colonia a nuestros días, México, El Colegio de México, Secretaría de Economía, 2010, pp. 285, 288.

Trijlllo Bolıo, Mario, Operarios fabriles en el Valle de México, 1864-1884, México, CIESAS, El Colegio de México, 1997.

TRUılıLo BoLIo, Mario, «La fábrica La Magdalena Contreras (1839-1910). Una empresa textil precursora en el valle de México», en Carlos Marichal y Mario Cerutti (comps.), Historia de las grandes empresas en México, 1850-1930, México, FCE, 1997, pp. 245-274.

VILlalobos, Liborio, Las obreras en el porfiriato, México, Plaza y Valdés, 2002. 


\section{Derechos de autor}

La revista Deusto Estudios Cooperativos es una revista de acceso abierto lo que significa que es de libre acceso en su integridad inmediatamente después de la publicación de cada número. Se permite su lectura, la búsqueda, descarga, distribución y reutilización legal en cualquier tipo de soporte sólo para fines no comerciales y según lo previsto por la ley; sin la previa autorización de la Editorial (Universidad de Deusto) o el autor, siempre que la obra original sea debidamente citada (número, año, páginas y DOI si procede) y cualquier cambio en el original esté claramente indicado.

\section{Copyright}

The Deusto Journal of Cooperative Studies is an Open Access journal which means that it is free for full and immediate access, reading, search, download, distribution, and lawful reuse in any medium only for non-commercial purposes, without prior permission from the Publisher or the author; provided the original work is properly cited and any changes to the original are clearly indicated. 